\title{
High-temperature fiber-optic Fabry-Perot interferometric sensors
}

\author{
Wenhui Ding, ${ }^{1}$ Yi Jiang, ${ }^{1}$ Ran Gao, ${ }^{1, a)}$ and Yuewu Liu ${ }^{2}$ \\ ${ }^{1}$ School of Optoelectronics, Beijing Institute of Technology, Beijing 100081, China \\ ${ }^{2}$ Key Laboratory for Mechanics in Fluid Solid Coupling Systems, Institute of Mechanics, Chinese Academy \\ of Science, Beijing 100190, China
}

(Received 27 October 2014; accepted 19 April 2015; published online 4 May 2015)

\begin{abstract}
A photonic crystal fiber (PCF) based high-temperature fiber-optic sensor is proposed and experimentally demonstrated. The sensor head is a Fabry-Perot cavity manufactured with a short section of endless single-mode photonic crystal fiber (ESM PCF). The interferometric spectrum of the Fabry-Perot interferometer is collected by a charge coupled device linear array based micro spectrometer. A high-resolution demodulation algorithm is used to interrogate the peak wavelengths. Experimental results show that the temperature range of $1200^{\circ} \mathrm{C}$ and the temperature resolution of $1{ }^{\circ} \mathrm{C}$ are achieved. (ㅇ 2015 AIP Publishing LLC. [http://dx.doi.org/10.1063/1.4919409]
\end{abstract}

\section{INTRODUCTION}

The igniter wire is an essential component in the electro explosive devices. The performance of the igniter wire is affected by the temperature which could be controlled by the driver current. In conventional methods, the temperature is measured by using a thermocouple or thermal resistor. However, these methods are unsuitable for the temperature measurement of an igniter wire which is as small as tens of micrometers in size. Fiber-optic temperature sensors exhibit outstanding advantages over conventional temperature sensors, such as small size, high precision, and wide measurement range. ${ }^{1}$ A large number of fiber-optic temperature sensors have been proposed and demonstrated. ${ }^{2,3}$ Among them, optical fiber sensors based on fiber Bragg grating (FBG) and interferometer are particularly attractive because they are insensitive with the light source intensity. The FBG is an ideal sensor for the temperature measurement due to its advantages such as wavelength coding, wavelength modulation, and intrinsic property. ${ }^{4}$ However, the FBG sensor performs poor stability in the hightemperature environment and the grating would be erased completely when the temperature exceeds $300^{\circ} \mathrm{C} .{ }^{5}$ Some interferometric temperature sensors can measure a wide range as high as $1600^{\circ} \mathrm{C} .{ }^{6,7}$ However, the configuration of these sensors is complex and the sensing element is an extrinsic sensor.

In this letter, we proposed a high-temperature fiber-optic Fabry-Perot interferometric (FFPI) sensor. The sensor is fabricated by splicing a segment of the endless single mode photonic crystal fiber (ESM PCF) to a single mode fiber (SMF28). The reflected spectrum of the FFPI is interrogated by using a micro spectrometer. Experimental results show that the temperature measurement range of the proposed sensor exceeds $1200^{\circ} \mathrm{C}$.

\section{OPERATION PRINCIPLE}

The fiber-optic temperature sensor is constructed by splicing a SMF with a short segment of the ESM PCF (SM-7.0-PCF,

\footnotetext{
a) Author to whom correspondence should be addressed. Electronic mail: bitjy@bit.edu.cn.
}

YOFC CO.), and the free end face of the PCF is cleaved and polished to a certain length $\mathrm{L}$. The operation principle of the sensor is shown in Fig. 1(a) and the microscope photograph of the sensor is illustrated in Fig. 1(b). At the fiber splice, a reflector $R_{1}$ with a reflectivity of $1.2 \%$ is formed because of the small difference in refractive index between the SMF and the PCF. The end face of the PCF acts as another reflector $\mathrm{R}_{2}$ due to the Fresnel reflection. Thus, an intrinsic Fabry-Perot interferometer is formed. The material of the EMS PCF is pure silica. The fiber has a $7 \mu \mathrm{m}$ diameter core, the average diameter of voids is $2.57 \mu \mathrm{m}$, and the average separation between the voids is $5.12 \mu \mathrm{m}$.

When the external temperature increases, the effective refractive index and the length of the PCF will be changed because of the thermo-optics effect and the thermal expansion effect. Thus, the change of the optical path difference (OPD), $\Delta \mathrm{d}$, can be expressed as

$$
\begin{aligned}
\Delta d & =2 L \cdot\left(\frac{d n_{\text {eff }}}{d T}+n_{e f f} \times \frac{d L}{L \cdot d T}\right) \cdot \Delta T \\
& =2 L\left(\sigma_{T}+n_{\text {eff }} \alpha_{T}\right) \Delta T,
\end{aligned}
$$

where $\Delta d$ is the change of the OPD, $n_{\text {eff }}$ is the effective refractive index of pure silica, $L$ is the physical length of the cavity, $\sigma_{T}$ is the thermo-optics coefficient, and $\alpha_{T}$ is the thermal expansion coefficient.

In the optical spectrum of the interferometer, with the increase of the temperature, peak wavelengths shift toward longer wavelength. When a peak wavelength changes from $\lambda_{0}$ to $\lambda_{n}$, the change of the OPD can be expressed as ${ }^{8}$

$$
\Delta d=k \times \Delta \lambda=k\left(\lambda_{n}-\lambda_{0}\right),
$$

where $k$ is the interferometric order of the fringe, which is an integer.

From Eqs. (1) and (2), the relationship between the change of a peak wavelength $\Delta \lambda$ and the temperature variation $\Delta T$ can be obtained as

$$
\begin{aligned}
\Delta \lambda & =\lambda_{n}-\lambda_{0} \\
& =\frac{2}{k} \times L \times\left(\sigma_{T}+n_{e f f} \cdot \alpha_{T}\right) \times \Delta T \\
& =\lambda_{0} \times\left(\sigma_{T} / n_{e f f}+\alpha_{T}\right) \times \Delta T .
\end{aligned}
$$




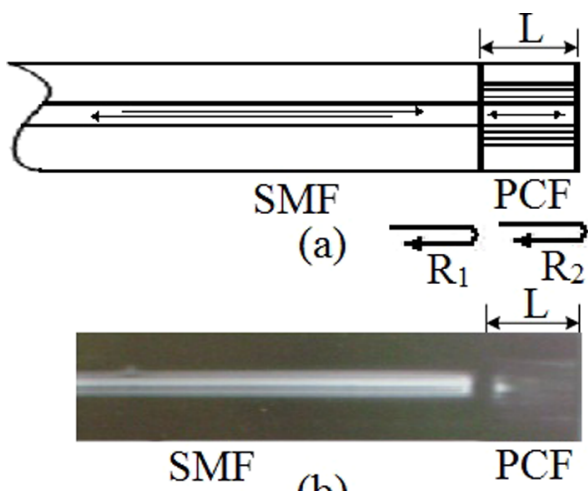

(b)

FIG. 1. (a) The operation principle. (b) The cross section of the ESM PCF.

For the sensor constructed in our experiment, the effective refractive index $n_{\text {eff }}$, the thermo-optics coefficient $\sigma_{T}$, and the thermal expansion coefficient $\alpha_{T}$ of the pure silica are given as $n_{\text {eff }}=1.4575, \sigma_{T}=1.45 \times 10^{-5} /{ }^{\circ} \mathrm{C}$, and $\alpha_{T}$ $=0.55 \times 10^{-6} /{ }^{\circ} \mathrm{C}$, respectively. ${ }^{9}$ The peak wavelength $\lambda_{0}$ is the initial peak wavelength. The sensor length $L$ can be roughly measured by using white-light interferometry (WLI). ${ }^{10}$ Thus, the wavelength shift, $\Delta \lambda$, is proportional to the temperature variation $\Delta T$. So, the change of the temperature can be measured by interrogating the peak wavelength in the optical spectrum of the FFPI.

\section{EXPERIMENTAL SETUP}

The experimental setup for interrogating the FFPI sensor is shown in Fig. 2. An unflattened amplified spontaneous emission (ASE) source with a wavelength covering $1525-1565 \mathrm{~nm}$ is used to illuminate a FFPI with a cavity length of $148 \mu \mathrm{m}$. The reflected spectrum of FFPI is collected by using a micro spectrometer (BaySpec FBGA-F-1525-1565), and the optical spectrum of the ASE source is shown in Fig. 3(a). Fig. 3(b) shows the reflected spectrum of FFPI. Due to the spectral profile of the unflattened ASE source, the peak wavelength around $1530 \mathrm{~nm}$ is significantly stronger than others.

The spectrum of the sensor is first sampled into a personal computer (PC). Due to the limitation pixels (512 pixels) and the narrow wavelength range (1513-1572 nm), the wavelength resolution of the micro spectrometer is only $0.115 \mathrm{~nm}$. Meanwhile, the spectrum of the sensor is a sine curve, or the peaks in the optical spectrum are not sharp; thus, there is much uncertainty in determining peak positions, which will reduce the measurement resolution. In our experiment, the resolution of the micro spectrometer is improved by a sub-division method. The sub-division method is developed by using the spline interpolation arithmetic. ${ }^{11}$

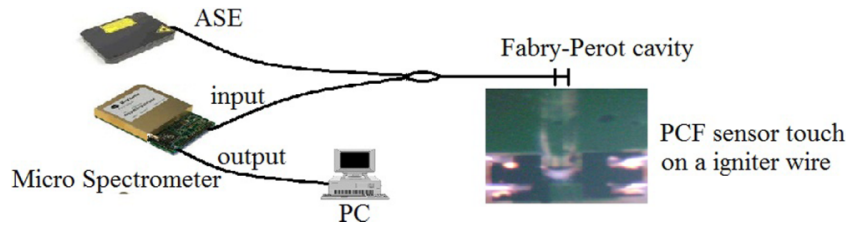

FIG. 2. Schematic diagram of the experimental setup.
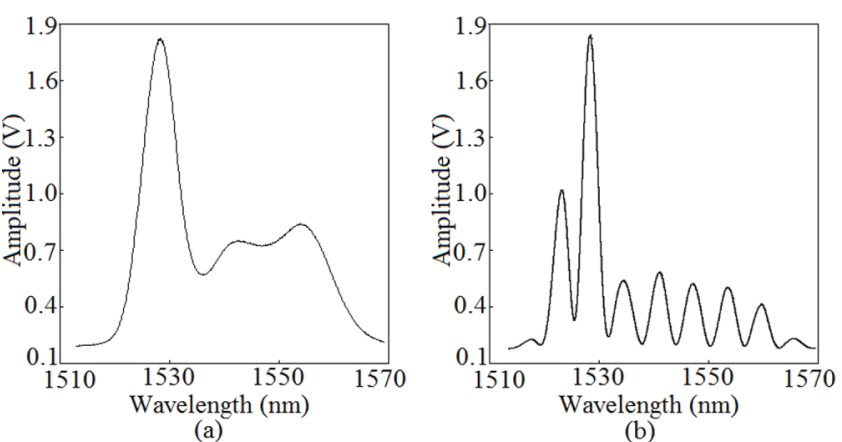

FIG. 3. (a) Optical spectrum of the ASE and (b) the reflected optical spectrum of the Fabry-Perot interferometer.

After the optical spectrum is sampled into the computer, we resample the pixels along the wavelength. The resampling interval of the wavelength is set to be $1 \mathrm{pm}$. So, the length of the resampled sub-pixels is 40000 for the wavelength range from 1525 to $1565 \mathrm{~nm}$. The sub-pixels with the length of 40000 are recovered by using spline interpolation arithmetic. Then, the wavelength interval of $1 \mathrm{pm}$ within the wavelength range of $40 \mathrm{~nm}$ can be obtained. The wavelength resolution of the micro spectrometer is improved from $0.115 \mathrm{~nm}$ to $1 \mathrm{pm}$.

\section{EXPERIMENTS AND DISCUSSION}

The temperature response of the sensor is experimentally investigated. The length of the sensor is $148 \mu \mathrm{m}$ of which the size is the same as that of the igniter wire $(\sim 100 \mu \mathrm{m})$. The small size of the sensor head is easy to be touched on the surface of the igniter wire. First, the sensor head was calibrated by placing the sensor into a muffle furnace. The temperature of the muffle furnace was adjusted from the room temperature $\left(17^{\circ} \mathrm{C}\right)$ to the $1200^{\circ} \mathrm{C}$. A peak wavelength with the change of the temperature was obtained, as shown in Fig. 4. The straight line in Fig. 4 is the linear fitting curve. The obtained linear fitting equation is $\lambda=0.01 \mathrm{~T}+1537$, which indicates the relationship between the wavelength shift and the temperature is $0.01 \mathrm{~nm} /{ }^{\circ} \mathrm{C}$.

Then, we measured the temperature of an igniter wire by touching the PCF sensor on the surface of the igniter wire, as shown in the inset figure of Fig. 2. When the current is zero, the temperature is measured as $31{ }^{\circ} \mathrm{C}$ by using a standard thermometer. Different driving currents were applied on the igniter

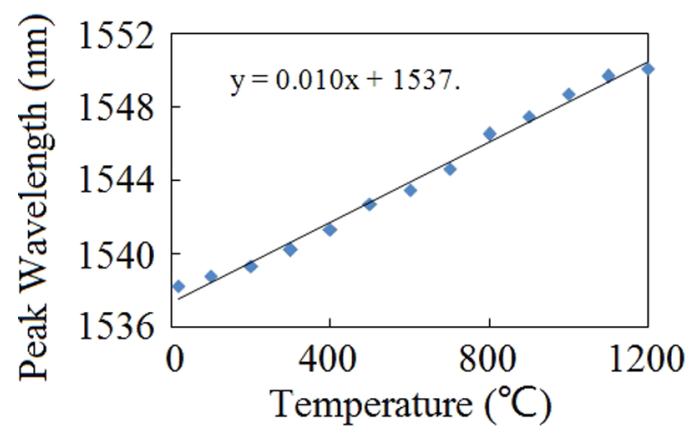




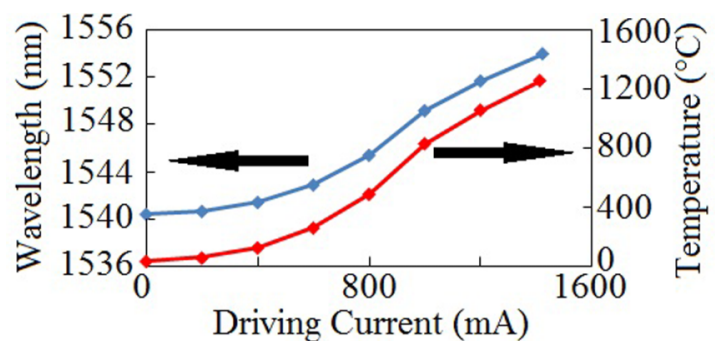

(a)

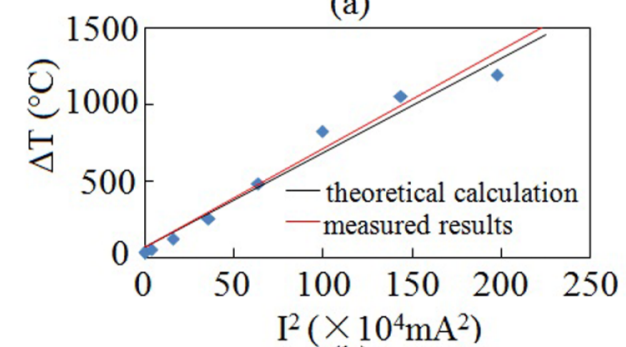

(b)

FIG. 5. (a) The measurement results of the temperature of the igniter wire and (b) the relationship between the temperature change $\Delta T$ and the square of the driving current $I^{2}$.

wire, and the temperature of the igniter wire is measured by interrogating the peak wavelength of the interferometric spectrum. The measured results are shown in Fig. 5(a). With the increase of the driving current, the temperature of the igniter wire is increased and the peak wavelength of the FP interferometer red-shift simultaneously. Because the temperature sensitivity of the sensor is $0.01 \mathrm{~nm} /{ }^{\circ} \mathrm{C}$, a temperature change of $1251{ }^{\circ} \mathrm{C}$ is calculated when the peak wavelength shift is $13.88 \mathrm{~nm}$. During the experiment, the fringe visibility of the optical spectrum has no distinct change even if temperature exceeds $1200^{\circ} \mathrm{C}$.

The relationship between the change of temperature and the driving current can be written as ${ }^{12}$

$$
\Delta T=C I^{2},
$$

where $C$ is a constant determined by the material and the size of igniter wire, $I$ is the driving current, and $\Delta T$ is the change of the temperature. Given that the constant $C$ is 6.46 in our experiment, which is calculated by using the parameters of the igniter wire. ${ }^{12}$ The measured results are compared with that calculated by using Eq. (4), as shown in Fig. 5(b). The measured results are in good agreement with that of theoretical prediction.

To test the stability and resolution of the system, a peak wavelength of the interferometer was measured for $1 \mathrm{~min}$ at room temperature. The experimental results are shown in Fig. $6(\mathrm{a})$, in which the peak wavelength is $1527.115 \mathrm{~nm}$ and the variation is \pm 5 pm during 6000 measurements. In the method without the sub-division, a temperature resolution of $11.5^{\circ} \mathrm{C}$ corresponding to the wavelength resolution of $0.115 \mathrm{~nm}$ is much lower than that of the sub-division method. Besides, a continuous test was performed when the temperature was changed from $400{ }^{\circ} \mathrm{C}$ to $480^{\circ} \mathrm{C}$ with a temperature interval temperature of $10^{\circ} \mathrm{C}$. The experimental results are shown in Fig. 6(b). A peak wavelength of the sensor shifts from 1534.730 to $1535.605 \mathrm{~nm}$ in 9 steps during the test. The change

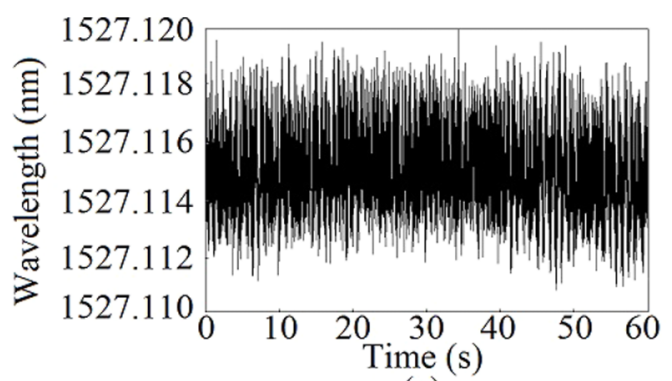

(a)

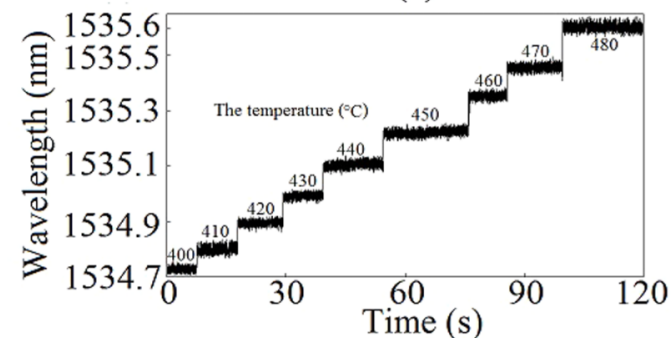

(b)

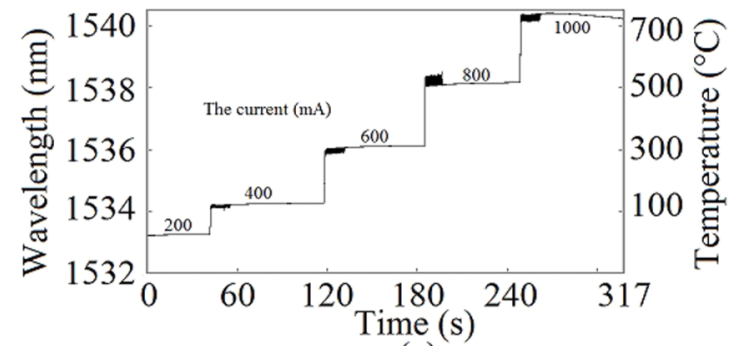

(c)

FIG. 6. The experimental result: (a) a continuous test at a fixed temperature, (b) a continuous testing when the temperature was changed from 400 to $480{ }^{\circ} \mathrm{C}$, and (c) a continuous testing when the current was changed from 200 to $1000 \mathrm{~mA}$.

of the peak wavelength is approximately $100 \mathrm{pm}$ in each step. Moreover, the interferometer was continuously tested by adjusting the driving current of the igniter wire from $200 \mathrm{~mA}$ to $1000 \mathrm{~mA}$ with a current interval of $200 \mathrm{~mA}$. With the increase of the driving current, the temperature of the igniter wire increases, which results in the red-shift of the peak wavelength. The experimental results are shown in Fig. 6(c) with the peak wavelength shift from $1533.250 \mathrm{~nm}$ to $1540.395 \mathrm{~nm}$ in 5 steps during the test. It is noted that the corresponding wavelength is also fluctuated at the beginning of each step, because the temperature of the igniter wire would become unstable in each staircase of current, as shown in Fig. 6(c).

\section{CONCLUSION}

In conclusion, a PCF based FFPI high-temperature sensor is presented and experimentally demonstrated. The optical interferometric spectrum of the FFPI is interrogated by using a micro spectrometer, and a sub-division technique is developed to improve the wavelength resolution. Experimental results show that the peak wavelength resolution of $10 \mathrm{pm}$ and the temperature sensitivity of $10 \mathrm{pm} /{ }^{\circ} \mathrm{C}$ are achieved. The reflected spectrum keeps stable even when the temperature exceeds $1200{ }^{\circ} \mathrm{C}$, showing that the FFPI based sensor exhibits a wide temperature measurement range. 


\section{ACKNOWLEDGMENTS}

This manuscript was funded by a National 863 Project (2015AA043504).

${ }^{1}$ T. Zhou, F. F. Pang, and T. Y. Wang, "High temperature sensor properties of a specialty double cladding fiber," Proc. SPIE 8311, 831100 (2011).

${ }^{2}$ B. Lee, "Review of the present status of optical fiber sensors," Opt. Fiber Technol. 9, 57-79 (2003).

${ }^{3}$ B. Culshaw and A. Kersey, "Fiber-optic sensing: A historical perspective," J. Lightwave Technol. 26, 1064-1078 (2008)

${ }^{4}$ B. W. Zhang and M. Kahrizi, "High-temperature resistance fiber Bragg grating temperature sensor fabrication," IEEE Sens. J. 7, 586-591 (2007).

${ }^{5} \mathrm{G}$. Brambilla, "High-temperature fibre Bragg grating thermometer," Electron. Lett. 38, 954-956 (2002).

${ }^{6}$ Y. Z. Zhu, Z. Y. Huang, F. B. Shen, and A. B. Wang, "Sapphire-fiber-based white-light interferometric sensor for high-temperature measurements," Opt. Lett. 30, 711-713 (2005).
${ }^{7}$ H. Xiao, J. D. Deng, G. Pickrell, R. G. May, and A. B. Wang, "Singlecrystal sapphire fiber-based strain sensor for high-temperature applications," J. Lightwave Technol. 21, 2276-2283 (2003).

${ }^{8}$ J. Zhang, H. Sun, Q. Z. Rong, Y. Ma, L. Liang, Q. F. Xu, P. Zhao, Z. Y. Feng, M. L. Hu, and X. G. Qiao, "High-temperature sensor using a Fabry-Perot interferometer based on solid-core photonic crystal fiber," Chin. Opt. Lett. 10, 070607-070609 (2012).

${ }^{9}$ T. Zhu, T. Ke, Y. J. Rao, and K. S. Chiang, "Fabry-Perot optical fiber tip sensor for high temperature measurement," Opt. Commun. 283, 3683-3685 (2010).

${ }^{10}$ Y. Jiang, "High-resolution interrogation technique for fiber optic extrinsic Fabry-Perot interferometric sensors by the peak-to-peak method," Appl. Opt. 47, 925-932 (2008).

${ }^{11}$ N. P. Sun, T. Ayabe, and T. Nishizaki, "Efficient spline interpolation curve modeling," in Third International Conference on Intelligent Information Hiding and Multimedia Signal Processing (IEEE, 2007), Vol. II, pp. 5962.

${ }^{12}$ S. G. Mei, T. Wang, F. Yin, J. F. He, C. Ruan, L. X. Wu, and T. Zhao, "Study on the induced current measurement in bridgewire EED," Microwave Opt. Technol. Lett. 50, 2833-2835 (2008). 